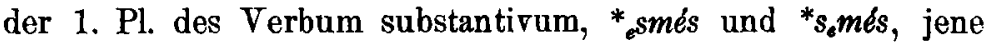
liegt den altpersischen, griechischen, keltischen und baltischslavischen, diese den italischen und germanischen Formen zugrunde. Vielleicht gab es daneben noch eine dritte, ${ }^{*} s m e ́ s$, die in ai. smás, av. mahi stecken könnte, jedoch können diese Formen auch durch Angleichung an die 3. Pl. entstanden sein.

Goes.

N. van $W i j k$.

\title{
Zur Wortzusammensetzung in den idg. Sprachen.
}

1. Die Stellung der Bahuvrihi im Kreis der Nominalkomposita.

Von den Bahuvrīhi-Komposita habe ich Grundr. 2, 87 (vgl. Kurze vergl. Gramm. $303 \mathrm{f}$.) gesagt, daß es sich bei ihrer Entstehung in der Hauptsache um die Verwandlung eines Substantivs in ein Adjektiv gehandelt habe; von der Bedeutung einer Substanz sei abgesehen worden, sodaß nur die der Substanz anhaftenden Qualitäten als Begriffsinhalt übrig geblieben seien.

Gegen diese Auffassung wandte sich Jacobi Comp. u. Nebens. $83 \mathrm{ff}$. und deutete die Bahuvrīhi als zu éinem Wort vereinigte Nebensätze. Dadurch, daß man den zugrunde liegenden Nebensatz (mit dem Verbum sein) auf seine nominalen Bestandteile reduziert habe, sei die Entstehung dieser Komposita ermöglicht worden. "Es entstand dadurch ein durchaus fester Typus, der das Muster abgeben konnte für die wenig zahlreichen Bahuvrïbi, die wir mit einem andern Verbum als dem des Habens oder Seins auflösen müssen, wie avó-dèva die 'Götter herbei(holend)', кaтó-ckıoc "Schatten herab(werfend)" "(S. 90).

Gegen Jacobi wiederum richtete sich Wheelers kleiner Aufsatz 'The So-called Mutation in Indo-European Compounds' in den Proceedings of the Am. Philol. Assoc., Vol. 34 (1903) S. LXVIII ff. Jacobi sei zwar, sagt Wheeler, auf der richtigen Fährte gewesen, er habe den Ursprung und die Entstehungsart aber doch nicht erkannt, weil er sich von den altindischen Bahurrïhi habe leiten lassen, statt, wie er hätte tun sollen, von den griechischen. Man müsse von dem nach der Verbaldiathese verschiedenen Charakter des Schlußglieds in griechischen Kom- 


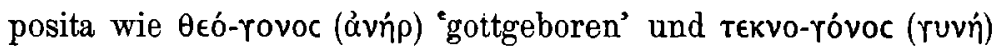
'kindergebärend" ausgehen. Der passivische Gebrauch des das zweite Glied von $\theta \in o ́$-rovoc bildenden Verbalnomens sei ursprünglich das Charakteristische für unsere Komposita gewesen. An diese passivische Vorstellung habe sich die des Versehen-

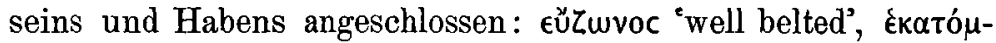

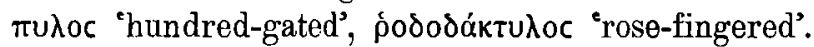

Daß auch Jacobi und Wheeler die Sache nicht erledigt haben, daß unsere Kompositionsklasse weder aus Nebensätzen, speziell aus Relativsätzen mit ausgelassenem Relativum, noch auch auf Grund des Gegensatzes von Aktivität und Passivität entwickelt sind, dürften die folgenden Erwägungen ergeben.

In den meisten Fällen, wo es zu einem Kompositum gekommen ist, ist dies so geschehen, daß die syntaktische Funktion der Wortgruppe, die Bedeutungsbeziehung, die zwischen ihr als Satzglied und andern Satzgliedern bestand, unverändert dieselbe blieb. Hierhin gehören verbale Komposita wie lat. cale-facio, ne-scio, con-ficio, von den nominalen und den Partikelkomposita solche wie lat. quis-quis ai. dáme-damē in jedem Hause' pró-pra

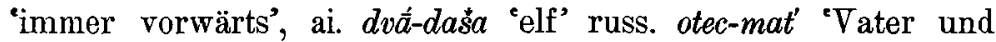

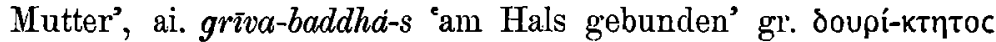
'durch Kampf gewonnen' útó- $\theta \in \tau 0 c$ 'untergelegt', ai. adharahanú-ş 'unterer Kinnbacken' rāja-putráts 'Königssohn' ati-dūro-s

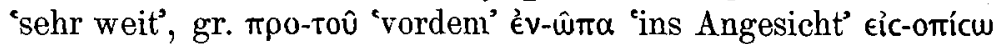
'künftighin', ai. na-hi 'gewiß nicht'. Allerdings hat in solchen Fällen der Kompositionsvorgang mitunter Änderungen in den Flexionsverhältnissen im Gefolge gehabt, z. B. ist öfters Endflexion für Binnenflexion eingetreten, wie bei poln. sztuka-mięsa 'ein Stück Fleisch(es)' (Gen. sztukamiesy) und lat. ipse $={ }^{*} i s-p s e$ (ipsum für eum-pse) u. dgl. Aber diese Neuerungen haben nicht die Geltung des ganzen Kompositionsgebildes im Satz berührt.

Von allen diesen Zusammensetzungen unterscheiden sich nun die Bahuvrihi durch das, was A. Aleksandrow Litauische Studien I: Nominalzusammensetzungen (Dorpat 1888) S. 110 nicht unpassend, wenn auch etwas äußerlich, ihren exozentrischen Charakter nennt. Bei ihnen ist nämlich zu den Vorstellungen, die durch die isolierten Kompositionsbestandteile ausgedrückt sind, und eventuell überdies zu der Bedeutungsmodifikation, durch die der Wortkomplex konventioneller Ausdruck für eine irgendwie einheitliche Gesamtvorstellung wurde, 
das Bedeutungselement hinzugekommen, da $\mathrm{B}$ der Begriffsinhalt der Zusammensetzung einem außerhalb stehenden Substantivbegriff als Eigenschaft beigelegt ist. Hierdurch erscheint, wie schon Justi sich ausdrückt (Zusammens. S. 118), das Subjekt nicht in dem Kompositum, sondern außer ihm liegend. Z. B. ai. kula-kula-s 'von Haus zu Haus (kula-kulam) gehend', rájjaputra-s 'einen König zum Sohn habend', anyá-rūpa-s 'andere Gestalt habend', bhúmi-grha-s 'die Erde als Haus habend', anantá-s 'endlos', ví-griva-s 'wem der Hals ab (abgeschlagen oder dgl.) ist'. Diesen Komposita gegenüber hätten demnach alle oben genannten, die nominalen wie die verbalen, es ozen tris ches Wesen.

Will man nun dem Ursprung der Bahuvrihi näher kommen, so hat man augenscheinlich alle exozentrischen Nominalkomposita als eine einheitliche Kategorie zusammenzunehmen. Bei ihnen ist die Beziehung der Glieder zueinander weit mannigfaltiger als bei den esozentrischen Nominalkomposita, weil viele von ihnen, wie man es gewöhnlich ausdrückt, 'auf Grund von Redensarten' entstanden sind. Es gehören hierhin besonders Eigennamen (Spitznamen u. dgl.) und eigennamenähnliche Wörter. Zunächst nenue ich die Imperativkomposita. Einzelsprachliche Beispiele dieser Gattung sind ai. jahi-stamba-s 'wer beständig an den Pfosten anschlägt' (jahi stambam 'schlag an den Pfosten an'), spätlat. Vincemalus (vince maloss), mhd. velle-walt 'Waldverderber' (Riesenname), nhd. Fürchte-gott, Trau-gott, Hebenstreit (heb-den-streit), sping-ins-feld, vergiss-mein-nicht nebst sauf-aus, kehr-aus, aslav. Mbsti-drugz čech. Msti-druh ('räche den Genossen'), vgl. Jacobi S. 57 f. 75 ff. Schon in uridg. Zeit ist so, wovon unten ausführlicher die Rede sein wird, der dem Arischen und dem

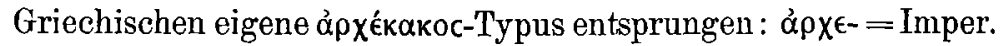
ă $\rho \chi \epsilon$. Fermer ai. ki $i$-rājan- 'wer ein schlechter König ist' auf Grund von 'was für ein König ist das?' u. dgl., wozu Analoga aus dem Griechischen bei Schulze KZ. 33, $243 \mathrm{f}$. Noch andere Beispiele verschiedener Art aus verschiedenen Sprachen sind: Ai. avó-dèva- (vácas) 'herunter die Götter holend, lockend', ahampūrvá- 'begierig der erste zu sein', kān-diš- 'flüchtig' auf Grund von 'nach welcher Richtung ( $k \bar{a}$ dišam) soll ich mich wenden?', yathä-tatha- 'wie es wirklich ist', mamasatyá-m 'wobei es sich um Mein und Dein handelt' etwa auf Grund von mama satyam 'mir (gehört es) wahrhaftig!', yadbhavişya- 'Fatalist' auf Grund von 'wer sagt: es kommt, was da kommt (yad bhavišyati)' (Whitney 


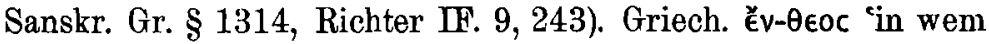

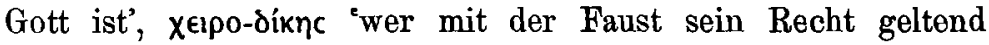

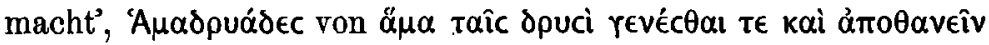

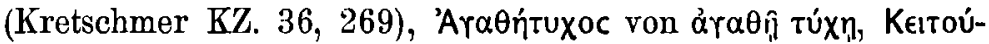
кєাтоC, Spitzname eines Grammatikers, der zu fragen pflegto

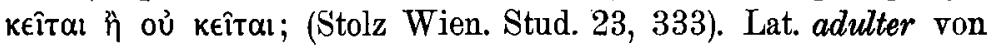
ad alteram se convertere, postprincipia Plur. 'was nach zurückgelegtem Anfang geschieht' von *post principio 'nach anfangs' wie post-modo 'nach sogleich, in Bälde' (Skutsch Fleckeisens Jahrbb. Suppl. 27, 97), virops 'quae iam virum (viro) opus habet', scherzhafte Bildung mit Anschluß an in-ops (Landgraf Arch. für lat. Lexikogr. 9, 440, Stolz a. a. 0. 314). Franz. un vive-lajoie 'einer, der immer vergnügt ist'. Nhd. je-länger-je-lieber, Pflanzenname, der gott-sei-bei-uns (Grimm D. Gr. Neudr. 2, 936 ff.). $\mathrm{Da}$ sie nicht allgemein zu den Bahuvrïhi gezogen werden, aber jedenfalls exozentrisch sind, mag hier ausdrücklich auch noch auf die zahlreichen präpositionalen Komposita von der Art der ai. upári-budhna-s 'über den Boden ragend', griech. éđi-raloc 'auf der Erde befindlich', lat. af-finis, ai. prá-napät lat. pro-nepos 'Urenkel', ursprünglich 'wer noch vor dem Enkel ist', verwiesen sein.

Ich stimme Jacobi darin durchaus bei, daß die Bahuvrihi als Bildungskategorie nicht aus einer oder mebreren der daneben stehenden Klassen von Nominalkomposita hervorgegangen sind, und daß ihre Bezeichnung als Mutata schief ist. Auch ist unzweifelhaft richtig, daß man zur Umschreibung der Bahuvrïhi meistens die Verba sein oder haben zu Hilfe nehmen kann. Aber daß diese Komposita in urindogermanischer Zeit einmal Nebensätze gewesen seien, ist mir von Jacobi ganz und gar nicht wahrscheinlich gemacht. Nimmt man, wie man muß, alle exozentrischen Komposita zusammen, so zeigt sich eine Mannigfaltigkeit der Beziehung der Zusammensetzungsglieder zueinander und des Verhältnisses der Glieder zu der zugrunde liegenden Gesamtrorstellung, die keineswegs danach aussieht, als sei die ganze Entwicklung von Nebensätzen von der Form, wie sie Jacobi annimmt, ausgegangen. Diese Tatsache ist freilich nicht ausschlaggebend. Denn man kann sagen: der Typus kam bei einfachen Verbindungen wie ai. anyó-rūpa- auf, und nachdem hier das Verbum sein aufgegeben und die nominale Flexion des Schlußglieds der Exponent der Eigenschaftsbedeutung geworden 
war, ging man weiter und schuf Komposita dieser Art auch von anderen Grundlagen aus, schuf z. B. Ėגк€xítwv auf Grund des

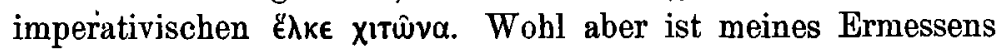
entscheidend der Umstand, daß die Nebensatzhypothese völlig überflüssig ist, daß die Klasse der exozentrischen Komposita vollkommen zu verstehen ist von der Annahme aus, daß hier das stattgefunden hat, was man Wortbildung durch Hypostasierung nennt.

Auf das Wesen des so bezeichneten Vorgangs muß ich kurz eingehen.

Ein ein- oder mehrgliedriger Bestandteil der Rede geht, wenn er, bei einer gewissen Erstarrung, mit einer Form andern grammatischen Charakters auf gleiche Linie gestellt ist, eine dieser Änderung des grammatischen Wertes entsprechende neue Verbindung im Satz ein. Dabei ist ein Doppeltes möglich. Die Hinüberleitung kann erstlich so vor sich gehen, daß nur eine neue syntaktische Verbindungsweise entsteht, nicht zugleich eine formantische Neubildung. Dahin gehört z. B. lat. frügì 'tüchtig', das der Dativ von früx 'Frucht, Ertrag' ist, wie er in der Wendung frugi est gebraucht war. Die Form wurde von hier in attributive Verwendung übergeführt, und man bildete z. B. servos frugi ${ }^{\text {cein }}$ tïchtiger Sklave, machte also frugi ohne formantische Neuerung zum Adjektiv. Ähnlich bewertete man Adverbia wie kasuelle

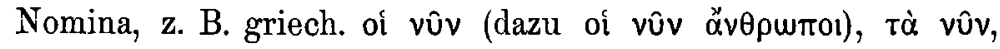
nhd. das hier, das dort, die dame in schwarz, lat. ad meridie, appetit meridie 'Mittag naht', Vokative wie Nominative, z. B. lat.

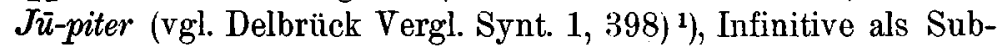
stantiva, z. B. griech. tò ảmoӨaveîv, u. dgl. Zweitens wird zugleich für das Formale des Wortes die Konsequenz gezogen: das Wort bekommt eine der neuen Verwendung im Satz entsprechende neue Flexionsendung. Bei Benutzung des Wortes als Adjektivum tritt oft eine dieser Funktion dienende Ableitungsendung an. Diese Änderungen geschehen natürlich alle nach mehr oder minder zahlreichen von älterer Zeit her bereit liegenden Mustern. Dabei bleibt nun die zugrunde liegende Formation entweder unverkürzt in ihrem Ausgang, die neue Flexionsendung tritt an diesen an, oder es wird ein 'Stamm' abstrahiert, der

1) Passend erläutert Delbrück diese Hypostasierung des Vokativs durch den Hinweis darauf, daß ein Erzieher bei Walter Scott von seiner Umgebung als der Domine bezeichnet wird. 
die Grundlage der neuen Form, bezw. Flexion bildet. Jenes z. B. in ein zufriedener mensch auf Grund von er ist zu frieden, nach der Art von ein bescheidener mensch neben er ist bescheiden, ein zues fenster auf Grund von das fenster ist zu, lat. meridiēs auf Grund des adverbialen meri-diē. Dieses z. B. in lat. postprincipia von *post principiō, spätlat. Vincemalus von Vince malös, ai. jahistamba-s von jahi stambam. Beispiele von Benutzung adjektivischer Ableitungsformantien sind: nhd. jetzig (mhd. ietze), desfallsig, zuen (ein zuenes fenster wie offenes), griech. Fi-dıc

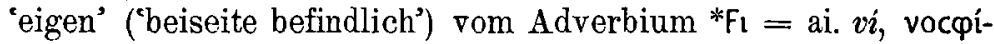
sıc 'entfernt' von vóçı, lat. merídiānus von meridiē, lit. musũjis 'unsrig' vom Gen. músu, dangujejis 'himmlisch' vom Lok. danguje, ai. šravấyya-s 'celebrandus' vom Inf. auf -āi, āstiká-s 'gläubig' von asti 'es ist', ki-yí-ş 'was begehrend' von kim 'was?' 1).

Hiernach sind die exozentrischen Nominalzusammensetzungen in urindogermanischer Zeit dadurch aufgekommen, daß Worte, die in einer gewissen kompositionellen Bedeutungsbeziehung zueinander standen, aber als Ganzes nicht den gram-

1) Zur Verdeutlichung des Wesens der hypostasierenden Wortbildungsweise mögen hier noch einige Beispiele von anderer Art folgen. Zunächst Beispiele für Pluralisierung, Dualisierung oder Singularisierung innerhalb von Kasussystemen. Ohne formantische Änderung wurde got. fadrein 'Elternschaft, Eltern' als Plural bewertet: pai fadrein wie bai sunjus; entsprechend wurde der aslav. kollektive Sing. bratzja 'fratres' (= $=\rho \bar{\alpha} \tau \rho^{i} \bar{\alpha}$ ) im Russischen Plural zu brat (daher Gen. brát'jev Dat. br'át' jam usw.) and schon im Urindogermanischen der kollektive Sing. *juga 'das Gejöche" Plural zu *iago-m (ai. yugd : yugám usw.); ebenso dürfte ai. amí 'illi' (wozu Instr. ami-bhis usw.) ursprünglich ein kollektives Fem. Sing. gewesen sein. Aksl. bratz-sestra 'Bruder und Schwester' ist dualisch geworden, daher bratzsestroma. Umgekehrt entstand der Sing. Schwaben auf Grund des Dat. Plur. Schwaben (mhd. zen Swäben); engl. gallows 'Galgen' war ursprünglich Plural, wurde dann aber als Sing. bewertet, was einen neuen Plur. gallowses hervorrief. Eine hypostatische Adverbialbildung ist z. B. lat. quotannīs: quot anni (sunt) bekam den Sinn 'in allen Jahren'

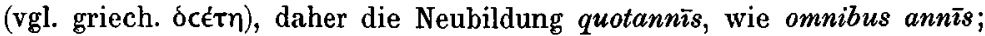
ebenso lit. kasmẽta 'alljährlich' auf Grund von kàs mẽts 'welches Jahr es auch sei' nach szĩ mëta. Entstehung verbaler Pluralformen : zur griechischen Partikel $\tau \hat{\eta}$ 'da! nimm' bildete Sophron den Plur. $\tau \hat{\imath} \tau \epsilon$; ebenso aksl. nate Plur. zur Partikel na 'da! nimm'; lit. dukite Plur. zu dưki 'gib' (-ki Par-

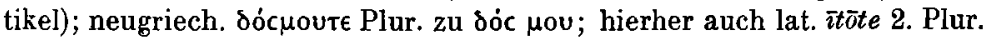

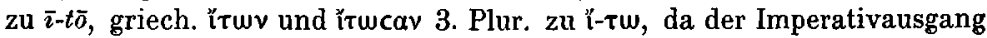
-tō $d$ wahrscheinlich ein Adverbium mit der Bedeutung 'dann, darauf’ gewesen ist. 
matischenCharakter eines eigenschaftsbegrifflichen Nomens hatten, in die Position solcher Nomina einrückten. Gleichwie die esozentrischen Komposita wie ai. adhara-hanu-ş "unterer Kinnbacken" rāja-putrá-s 'Königssohn', reichen die exozentrischen wie anyárūpa-s 'andere Gestalt habend' rájja-putra-s 'einen König zum Sohn habend' hiranya-hasta-s 'goldhändig' pätra-hasta-s 'ein Gefäß in der Hand habend' mit der Gestaltung des Vorderglieds in eine Sprachperiode hinauf, in der das Kasussystem noch nicht ausgestaltet war. Die eigenschaftsbegriffliche Beziehung der Bahuvrīhi war aber damals nicht bloß durch den Zusammenhang, durch die Stellung zu den andern Satzgliedern gegeben, etwa wie im Lateinischen in dem Satze nuntii nobis tristes venerant ex ante diem III Non. Iun. usque ad pridie Kal. Sept. der grammatische Charakter der Datumbezeichnung nur aus den vorgesetzten Präpositionen $e x$ und $a d$ erhellt. Sie war wenigstens in einem großen Teil der Fälle auch durch die Betonungsweise ausgedrückt. Denn bekanntlich wird die Betonungsdifferenz zwischen ai. rája-putra-

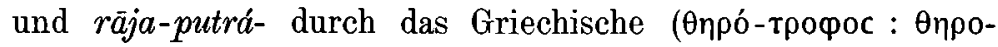
трó甲оc) und das Germanische (ags. fyder-féte 'vierfüßig' urgerm. *fíbur-, wie ai. cátušspad-) als urindogermanisch erwiesen. Diese selbe Anfangsbetonung anch in ai. vi-grīva-s 'dem der Hals ab ist', avṓ-dêva-s 'herunter die Götter holend', ihá-citta-s 'mit hierher gerichtetem Sinn', ánu-vrata-s 'nach der Anweisung verfahrend',

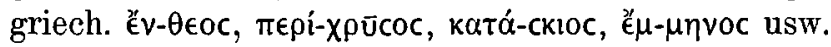

Die eine engere apperzeptive Einheit bildende Gruppe konnte also ursprünglich ohne weiteres, sei es vollständig, sei es mit Ausscheidung unwesentlicherer Elemente, als ein eigenschaftsbegriffliches Wort, als Name für ein Wesen oder adjektivisch, in den Satzzusammenhang eingestellt werden. Zwischen dieser ursprünglichen Form und der späteren, die uns überliefert ist, war das Verhältnis etwa dasselbe wie zwischen griech. $\tau \dot{\alpha}$

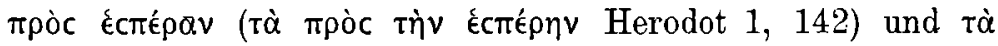

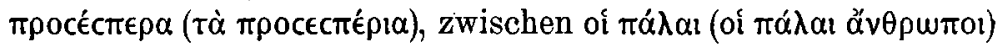

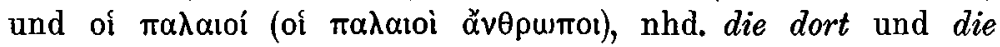
dortigen ${ }^{1}$ ). Als die exozentrischen Komposita, wie die anderm Nominalkomposita und überhaupt die Adjektiva und Substantiva, ihre Kasusflexion bekamen, ergab sich diese aus der jedesmaligen

1) Vgl. auch russ. massivnaja, dubovyja i iz cernago dereva kresla 'massive, eichene und aus schwarzem Holz (d. i. Ebenholz) Sessel', das wegen des gleichstellenden $i$ interessant ist.

Indogermanische Forschungen XVII. 
Natur des Ausgangs des Schlußglieds. Wie die Fälle zu beurteilen sind, wo konsonantische Stämme mit o-Flexion erscheinen, wie ai. prá-pada-m 'was vorn am Fuß ist, Fußspitze', adhas-padá-

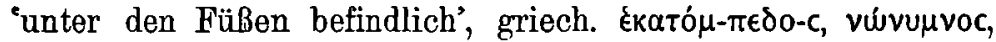

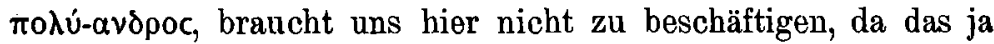
auch bei den esoterischen Nominalkomposita vorkommt. Immerhin mag nicht unangemerkt bleiben, daß Eintritt in die o-Flexion auch sonst gerade bei hypostatischen Gebilden von ältester Zeit an vorkommt: vgl. z. B. *upero-s (ai. upara-s lat. superus) zu *uper

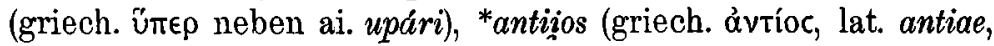
ahd. N. andi 'Stirn') zu *anti (griech. òvti); lat. meus = *meio-s aksl. mojb zum possessiven Lokat. *mei ${ }^{*}$ moi (ai. $m \bar{e}$ ); ${ }^{*}$ de $\hat{k}$ cmmo-s lat. decimus, *neunno-s lat. nönus zu *dekm, *neun (dies decimus war ursprünglich nur etwa 'der Tag Numero Zehn').

Ein jüngerer, aber ebenfalls schon uridg. Vorgang war, daß adjektivisches Wesen unserer Komposita durch das Formans -iio- gekennzeichnet wurde: ai. ipa-mäsya-s 'allmonatlich' griech.

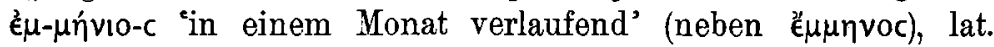
$\ddot{e}$-gregius, got. uf-aipeis 'unter einem Eide stehend', aksl. bez-umljb 'ohne Sinn', griech. ó $\mu 0-\pi \alpha ́ \tau p l o c ~ a i s l . ~ s a m-f e d r$ 'den gleichen Vater habend' (neben ó $\mu 0-\pi \alpha ́ \tau \omega \rho$ apers. hama-pitar-), ai. mádhu-hastya-s 'Süßigkeit in der Hand haltend', lat. acu-pedius bi-pedius, ags. án-féte 'einfüßig', arm. srb-azan -ic 'von reiner Art, heilig' (vgl. Meillet Mém. 11, 390 f.), lit. tri-rạzis 'dreizinkig'. Dieses adjektivierende -iio- erscheint seit derselben Zeit auch bei den Simplicia, z. B. ai. pitrya-s (pitriya-s) griech. $\pi \alpha^{\prime} \rho 10 c$ lat. patrius ${ }^{1}$ ).

1) $\mathrm{Da} *$ antizio-s von *anti ausgegangen ist (s. o.), *alio-s ‘alius' von *ali- (lat. ali-ter), *medhio-s 'medius' von *me-dhi 'inmitten' (vgl. $\mu \epsilon-\tau \grave{\alpha}$ ),

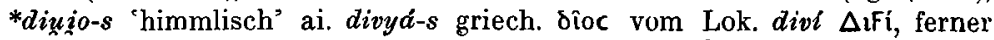

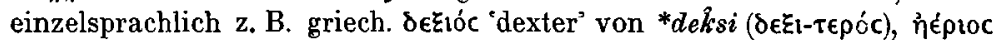

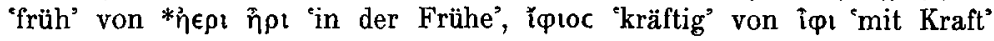
u. dgl. (Sommer IF. 11, 1 ff., Verf. Gr. Gr. ${ }^{3}$ 180), so ist es schwer, den Gedanken abzuweisen, das ganze urindogermanische der Adjektivbildung dienende -ijo- habe davon seinen Ausgang genommen, daß Formen auf $-i$, die zu adjektivischer Funktion im Satz gelangt waren, in die o-Deklination eingestellt wurden. Die zugehörigen Formen auf $-i-s$, wie av, $\bar{a} h u^{i}$ ri-s $s$ "von Ahura herrührend, göttlich' (Gen. ahurōis und ahüiryehe), mazdayasni-š 'den Mazdayasnern zugehörig' (Bartholomae Grundr. d. iran. Philol. 1, 44. 105), arm. srb-azan -if, an-gorc -ic (Meillet a. a. 0.), lat. ex-somnis, im-berbis, ir. do-chenéuil zu cenél, s-aidbir 'reich' zu adbar adbur (Stokes KZ. 38, 464f.), lit. tri-rặzis, minkszt-ga lvis 'schwachköpfig', verhalten sich dann zu den Formen auf -iigo-s (ai. -iyas, griech. -ıoc usw.) so, daß die Kasusbildung 
Die exozentrischen Nominalkomposita blieben im wesentlichen eine auf eignen Füßen stehende Bildungskategorie. Doch kamen sie mit andern Nominalkomposita in Berührung und mischten sich mit ihnen. Ein großer Teil von ihnen hatte nämlich seit uridg. Zeit Komposita neben sich, deren Glieder dieselben Wörter oder ganz gleichartige Wortarten waren, die aber eine esozentrische Bedeutung hatten, z. B. räja-putra-s

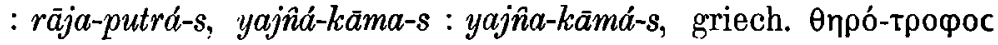

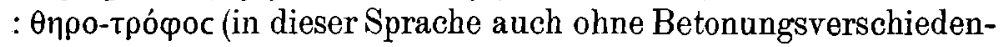
heit $\kappa \alpha \lambda \lambda i-\pi \alpha$ kc 'schöne Kinder habend' und 'schönes Kind'). Da nun die exozentrischen gegenüber den esozentrischen das Plus des Eigenschaftsbegriffs hatten, so konnten manche leicht als auf der andern Klasse beruhend und von ihr ausgegangen erscheinen. Es geschah dann dasselbe, was man anderwärts z. B. bei den Komparationsformen beobachtet: bekanntlich waren Komparativ und Superlativ ganz unabhängig vom sogenannten Positiv ins Leben getreten, die systematische Gruppierung, bei der der Positiv als Grundform erscheint, war eine sekundäre Entwicklung. Freilich ist nur von wenigen Bahuvrīhi wirklich zu beweisen, daß sie erst im Anschluß an schon vorhandene gleichstämmige esoterische Komposita ins Leben getreten sind. Es sind solche wie ai. rätridiva-m, substantiviertes Neutrum, 'Zeitraum von Tag und Nacht', auf Grund von rätrį-divā -divam 'bei Tag und bei Nacht' (anderes der Art bei Richter IF. 9, 240), kulakula-s, auf Grund von kula-kulam, womit sich z. B. lat. svāvior rergleichen läßt, in das ein Bildungselement des Positivs eingegangen ist (vgl. sváaìyas- ìdíuv).

Mit dieser sekundären Anlehnung an Komposita esoterischen Charakters hat, wie noch betont werden muß, der mangelhafte

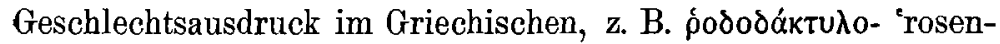

teils direkt von der Grundform auf $i$ aus, teils durch Überführung dieser in die o-Deklination geschah (dieses Verhältnis ist wohl nicht auch für lat. alis neben alius anzunehmen, s. Sommer Lat. Laut- u. Formenl. $367 \mathrm{f}$.). Die regelmäßjige Durchführung der o-Deklination durch alle Kasus in allen Wörtern, wie sie im Griechischen und im Germanischen vorliegt, wäre somit einzelsprachliche Neuerung gewesen. (Die Bemerkungen Schulzes Lat. Eigenn. 435 über das adjektivbildende -izo- -ijo- scheinen mir nur zum Teil richtig zu sein.) - Daß auch die Verbaladjektiva des Typus orroc (Grundr. 2, 117) von Bildungen auf $-i$ ausgegangen sind, darauf scheinen got. brüks aus *brüki-z u. dgl. Formen zu weisen (vgl. Walde Germ. Auslautges. 139). 
fingerig', trotz -o- auch für das Femininum, $\chi \rho \bar{u}$ cokó $\mu \eta-$ 'goldhaarig', trotz - $\eta$ - auch für das Maskulinum, nichts zu schaffen. Er beruht allerdings auf dem substantivischen Ursprung dieser Komposita. Aber es hat keine Umwandlung eines Determinativums in ein Bahuvrihi stattgefunden, sondern fododákтu入oc bedeutete von vornherein, halb namenartig, ein Wesen, dessen Eigenschaft ist, da $B$ es rosige Finger hat, $\chi \rho \bar{v}$ cokó $\mu \eta$ - ein Wesen mit goldigem Haar, gleichwie nhd. das krummbein ein Wesen mit krummem Bein, der freigeist ein Wesen mit freiem Geist, der dreifuss ein Wesen mit drei Füßen, ai. mahábāhhuss̆ und griech.

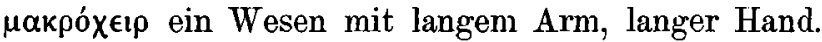

Es hat sich uns ergeben, daß zwischen den sogen. Bahuvrīhi und den übrigen exozentrischen Nominalkomposita nicht zu unterscheiden ist. Man wird daher gut tun, in der allgemeinindogermanischen Grammatik den Namen Bahuvrīhi entweder ganz fallen zu lassen und nur von exozentrischen oder, was ebenfalls anginge, hypostasierenden oder hypostatischen Nominalkomposita zu sprechen, oder aber dem Namen Bahuvrīhi eine erweiterte Anwendung zu geben und ihn gleichbedentend mit exozentrischem Kompositum zu gebrauchen.

\section{Der $\alpha \rho x \in ́ x \alpha$ кос-Typus und Verwandtes.}

Am wenigsten unter den exozentrischen Komposita sind bis jetzt die drei Bildungstypen entwicklungsgeschichtlich auf-

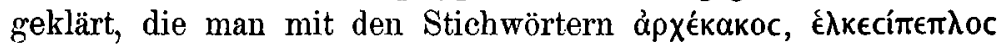
und (ai.) vidádvasuṣ benennen kann. Gemeinsam ist ihnen die Vorausstellung eines verbalen Bestandteils, der den zweiten, nominalen, regiert. Über sie, im ganzen oder teilweise, ist in letzterer Zeit gehandelt worden von Jacobi Compositum und Nebensatz, mir Griech. Gramm. ${ }^{3} 168$ f., Ber. d. sächs. Gesellsch. d. Wissensch. 1899 S. 195 ff., Delbrück Vergl. Synt. 3, 174, Foy KZ. 37, 544 f., Johansson Nordiska Studier (Uppsala 1904) S. 474 [Dittrich Zs. f. rom. Phil. 22, 317 ff. 323 f.].

Ich gebe zunächst Beispiele.

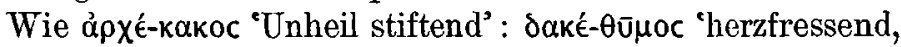

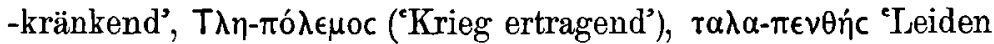
ertragend', é $\lambda \in \lambda \hat{i}[\mathrm{k}]-\chi \theta \omega v$ 'die Erde erschütternd', av. vinda- $x^{v} a r^{\curvearrowright} n a-$

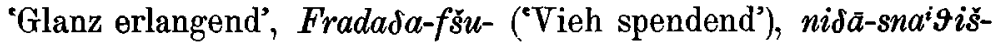
'die Waffen niederlegend', is $s^{\circ} x \check{s} a 9 r a-$ 'das Reich beherrschend', ai. dídy-agni- 'feuerleuchtend'. Unsicher ist ai. sthátrašman- 
RV. 5, 87, 5 von den Maruts: 'feststehend mit den Zügeln'; gewöhnlich wird übersetzt 'mit festen Zügeln' 1).

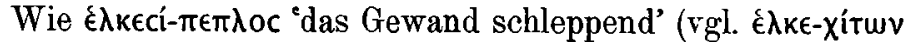

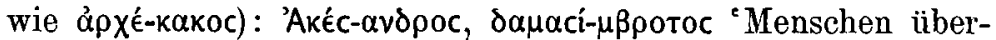

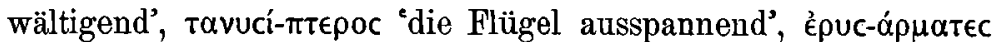

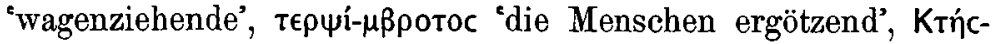
avdpoc, ai. dátti-vāra-s 'Gaben spendend' (vgl. $\Delta w c i-\varphi \rho \omega v)$, Rantidëva-s ('die Götter erfreuend'), vitt-hōtra-s 'Opfergaben verschaffend', rity-àp- 'wasserströmend'.

Wie vidád-vasu-ş 'Güter gewinnend' : Bharád-vãja-s ('Labung bringend'), dhārayát-kavi-šs 'Weise erhaltend', av. vanat-pəšana'Schlachten gewinnend', Dãrayat-ra9a- ('Wagen besitzend'). Für die Auffassung des zweiten Gliedes, als akkusativisch vom ersten Glied abhängig, ist interessant der av. Name eines Genius Frādatvispamhujyāiti-šs, 'jegliches zu gutem Leben dienende Mittel fördernd', dessen Schlußteil die Akkusativverbindung vīspam hujy $\bar{a}^{i} t \bar{t} m$ war. Nur mit -t- (nicht -at-) als Endformans des ersten Gliedes av. vikarot-uštāna- 'das Leben beeinträchtigend' (nach Bartholomae Grundr. d. iran. Phil. 1, 149).

Grundsätzlich hat man zunächst zu sehen, ob eine Deutung möglich ist, die diese Kompositionstypen nicht anders entwickelt sein läßt, als auch sonst nominale Zusammensetzungen im Kreis der idg. Sprachen zustande gekommen sind. Aber nur für die

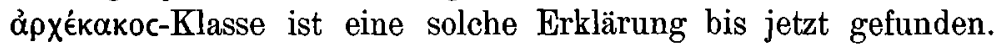
Es ist die, nach der der erste Bestandteil eine Imperativform

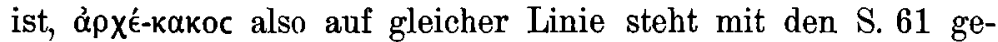
nannten ai. jahi-stamba-s, spätlat. Vince-malus, nhd. Fürchte-gott, cech. Msti-druh. Gegen diese Ansicht ist bis jetzt noch kein irgend stichhaltiger Einwand erhoben worden, und nur sie wird den überlieferten Tatsachen wirklich gerecht. Denn nur bei ihr erklären sich zwanglos das $\epsilon$ von ảoxé-kakoc und die Torausstellung des verbalen Bestandteils. Bei den Verbalstämmen ohne thematischen Vokal erscheint auch im Ar. noch die Imperativ-

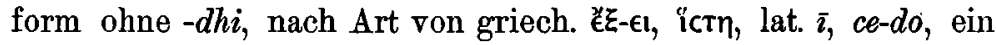
Zeichen, daß schon früh der imperativische Sinn sich verdunkelte (vgl. nhd. ein tu-gut mit dem danach gebildeten tu-nicht-gut, wo ebenfalls kein Imperativ mehr empfunden wird).

1) Unwahrscheinlich ist, was Ludwig Rigveda 5, 254 vermutet: 'die die Zügel des Festen besitzen', was so viel als 'die das Feste stürzen können'sei. 
Diese Auffassung der ả pxékakoc-Bildungen hätte wahrscheinlich weit mehr Anklang gefunden, als sie gefunden hat, wenn nicht die beiden andern Kompositionstypen daneben stünden, bei denen die Annahme eines imperativischen Anfangsteils bisher unmöglich erschienen ist. Man möchte natürlich gerne das funktionell Gleiche von gleichem Gesichtspunkt aus beurteilen,

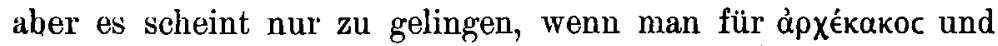
Genossen die Imperativtheorie fahren läßt ${ }^{1}$ ). Nun kommt freilich für den $\varepsilon \lambda \kappa \in c i ́ \pi \epsilon \pi \lambda o c-$ und den vidádvasu-Typus a priori auch die Möglichkeit in Betracht, daß sie vorn zwar keine imperativischen Formen enthalten, sondern nominale, aber in ihren ältesten Exemplaren eine Umdeutung nach Maßgabe von à $\rho \chi \hat{f}^{-}$ какоc-Komposita erfahren haben. Aber auch dieser Weg der Erklärung ist bis jetzt mit Erfolg nicht beschritten worden.

Man muß zugeben, daß der formantische Charakter der Vorderglieder des є̇ $\lambda \epsilon \in c i ́ \pi \epsilon \pi \lambda$ oc-Typus zunächst auf Verbalabstrakta oder Nomina actionis mit dem Formans - $t i$ - hinweist, Substantiva, die seit urindogermanischer Zeit auch als Nomina agentis verwendet werden konnten (Grundr. 2, 276. 431). Es ist aber bis jetzt unbeachtet geblieben, daß diese Anfangsglieder in die Verbindung mit den von ihnen regierten Schlußgliedern trotzdem als Formen imperativischen Sinnes eingegangen sein können. Sie können nämlich konjunktivisch-imperativische Infinitive gewesen sein.

Die $t i$-Substantiva sind bei der Infinitivbildung beteiligt im Arischen, z. B. pitáyēe 'zu trinken' av. fraoxtayaē[-ča] 'herzusagen'

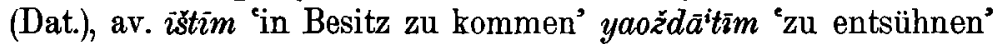
(Akk.), darštōiš 'zu sehen' (Gen.), taródititi 'despicere' apayeitī 'wegzunehmen' àn $\partial^{i} t \bar{\imath}$ 'zu atmen' (Instr.) (s. Delbrück Ai. Synt. 424, Vergl. Synt. 2, 450, Bartholomae BB. 15, 238 ff., Grundr. d. iran. Phil. 1, 144 ff.) und im Baltisch-Slavischen, z. B. lit. düti aksl. dati. Konjunktivisch-imperativisch gebraucht erscheinen 'Infinitivformen' der verschiedensten Bildungsart im Arischen wie in den europ. Sprachen: die Satzaussage, zu der der Infinitiv von Haus aus gehörte, wurde nicht ausgesprochen, sondern nur hinzuempfunden, wodurch der Infinitiv mit den adhortativen Formen des Verbum finitum auf gleiche Linie kam (Delbrück Vergl.

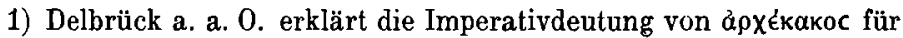
die wahrscheinlichste und fügt hinzu: "Über die beiden anderen Typen ( $€$ $\kappa \in c i \pi \in \pi \lambda$ oc und vidadvasu-) möchte ich mich nicht aussprechen". 
Synt. 2, 453 ff., Verf. Kurze vergl. Gr. 604). Sollten also nicht die $t i$-Formen unserer Komposita ursprünglich solche imperativische Infinitive gewesen sein? Der adhortative Infinitiv hat sich in modernen Sprachen verschiedentlich, besonders in der Alltagsrede, von neuem erzeugt (z. B. nhd. aufstehn!, hergeben!, den fuss vorsetzen!), und so ist es wenig kühn, anzunehmen, daß Nomina actionis auch schon in urindogermanischen Zeiten zu solcher Verwendung gekommen seien.

Es wäre hiernach die sogenannte Stammform, auf $-t i$, infinitivisch geworden ${ }^{1}$ ).

Oder soll man unsere Infinitivformen auf $-t i$ Lokative von $t$-Stämmen nennen? - $t i$ - und $-t$ - wechselten seit urindogermanischer Zeit als Stammauslaut bei den femininischen Abstrakta. Die $t$-Flexion ist repräsentiert durch ai. stút- av. stüt- 'Preis' (neben ai. stuti-ş), ai. vrgt- 'Begleitung', eigentlich 'Einzäunung' (neben

1) Hierzu bietet vielleicht der ar. Imperativ auf $-t u$ ein Analogon. Gegen die von Vielen angenommene Hypothese, daß bháatu, bharantu Injunktivformen mit angehängter Partikel $u$ seien, macht Delbrück Vergl. Synt. 2, 35̃7 geltend, daß die tu-Form nur positiv vorkommt, während man sie, wäre sie Injunktiv, vorzugsweise im Verbot $\mathrm{zu}$ erwarten hätte. Dieser Einwand ist sehr berechtigt. Es könnte also Gaedicke Der Acc. im Veda 254 Fußn. recht haben, der vermutet, hántu sei die Stammform der Infinitive hántos, hantave, hantum. Es gehörten dann noch z. B. dätu und dátōoş dátavē dâtum, êtu und êtōos êttum engstens zusammen. Durch Assoziation des Ausgangs $-t u$ mit den Personalendungen - $t i$ - $t$ kam man einerseits $\mathrm{zu}$ den Pluralformen ghnántu, ddntu, yántu (wie man im Griechischen $\varphi \in \rho o ́ v \tau \omega$ zu $\varphi \in \rho e ́ \tau \omega$, im Lateinischen vehuntō zu vehitō schuf), anderseits zur Ausbreitung auf die verschieden gestalteten Tempusstämme, z. B. bháratu nebst bhárantu, şrottu nebst şrnvántu usw. Alle diese Neuerungen hätten sich wahrscheinlich schon in urarischer Zeit abgespielt, weil das Iranische dieselben $t u$-Formen aufweist wie das Indische, z. B. av. baratu barantu neben jantu. Man darf überdies freilich auch an Zerlegung von bháratu in Imperativ bhára + Partikel tu (tú im RV. bei Aufforderungen üblich, s. Grafmann s. v., Delbrück Ai. Synt. $517 \mathrm{ff}$;; verwandt ist got. $\not a u$ bau-h, ahd. $d o h$ 'doch') denken. Die Partikel wäre im Lauf der Zeit bedeutungslos geworden, wie in urindogermanischer Zeit

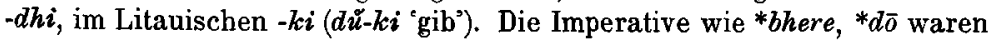
von Haus aus nicht auf den Gebrauch für die zweite Person beschränkt (Grundr. 2, $1323 \mathrm{f}$.), und die Einschränkung von bharatu, dattu auf die dritte Person wäre wiederum durch engere Assoziation von $-t u$ mit $-t i-t$ bewirkt worden und hätte in der Gebrauchsbegrenzung von griech. $\varphi \in \rho \hat{k}-\tau \omega$ ein Analogon. Von einer irgend zuverlässigen Deutung des ar. tu-Imperativs kann hiernach heute noch nicht die Rede sein. 
vrti-ş), sam-it- 'feindliches Zusammentreffen' (neben sám-iti-ş̌), av. $x$ šnūt- 'Befriedigung', ai. sarit- sravát- pravát- vahát- 'Strom, Fluß' (über pravát- s. Pischel-Geldner Ved. Stud. 2, 63 ff.), lat. teges, seges, got. mitaps (Stamm mitad-) 'Maß'. Vgl. auch den Wechsel zwischen -ti- und $t$ - bei dem Wort für die Nacht ai. nákti-ş lit. naktìs usw. neben griech. vúz vukTóc usw. und bei den Zahlabstrakta wie ai. dašati-ş aksl. desętz usw. neben ai. dašátaksl. Plur. desęt-e usw.

Wir kommen auf diese, für die Erklärung der ersten Glieder der $\varepsilon \lambda \kappa \epsilon c i \pi \epsilon \pi \lambda$ oc-Formen als imperativische Infinitive unwesentliche Frage wegen der vidádvasu-Formen, die formantisch engstens mit ihnen zusammengehören könnten, unten noch einmal zurück. Hier sei jedoch gleich noch folgendes bemerkt. Av. frā $\delta a t i[-\grave{c} a]$ in yt. 6, 1 ist wahrscheinlich Infinitiv ('zu fördern'). Von Bartholomae wird es als Lokativ eines Nomen actionis frājat-angesprochen (Grundr. d. iran. Phil. 1, 146), während Grégoire KZ.35, $100 \mathrm{f}$. die Auffassung als Instr. eines Stammes fräjati- vorzieht. Ich weiß eine Entscheidung nicht zu treffen. Waren also vielleicht die infinitivischen Formen auf $-t i$ einzelsprachlich in nichtimperativischer Bedeutung nicht mehr im Gebrauch, so vergleicht sich das damit, daß die altindischen Formen stôsssi, jéşşi, sátsi usw. und av. döiš̃, die lokativische Infinitive von $s$-Stämmen sind, ebenfalls nur noch in der imperativischen Anwendung des Infinitivs erscheinen (Neisser BB. 20, 70 ff., Bartholomae IF. 2, 271 ff., Delbrück Vergl. Synt. 2, 447). Ebenso hat der lateinische Imperativ sequimini, den man wohl richtig als dativischen Infinitiv betrachtet (griech. - $\mu \in v a l$ ), keine andern Infinitivfunktionen daneben behauptet.

Im Griechischen stand -cl- ursprünglich auch vor vokalisch

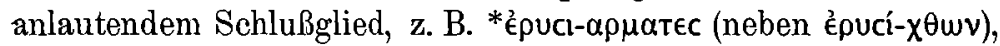
wie ai. rityà $p$ - d. i. ritit-ap-. Als nun, in urgriechischer Zeit, durch die bekannte Übertragung der Auslautelision kurzer Vokale in die Kompositionsfuge z. B. kam, entstanden auch épuc-á $\rho \mu \alpha \tau \in c, K \tau \eta \dot{c}-\alpha v \delta \rho \circ c$ usw. Daß es der verbale Charakter des Vorderglieds war, der die Aufgabe des -1 veranlaßte, wird dadurch bestätigt, daß die ti-Abstrakta, wie überhaupt nominale $i$-Stämme, sonst diesen Stammauslaut vor rokalisch anhebendem Schlußglied festhielten, z. B. craci- $\alpha \rho \chi 0 c$

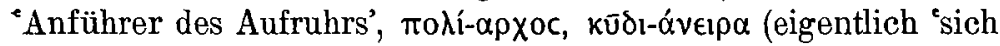
auszeichnende Männer habend'), s. Ber. d. sächs. Ges. d. W. 1899 
S. 1.97 ff. ${ }^{1}$ ). Dieser verbale Charakter war es auch, der die Assoziation mit den c-Tempora hervorrief, wodurch Formen wie

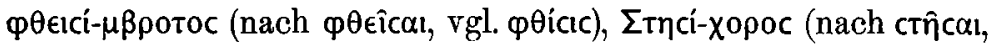

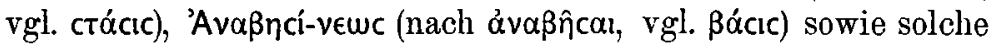

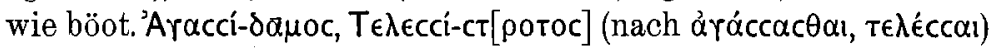
entsprangen. Dieser Anschluß an die c-Tempora ist zwar auch außerhalb der $\epsilon \lambda \kappa \in c i \pi \epsilon \pi \lambda o c-K o m p o s i t i o n ~ d e n ~ t i$-Abstrakta nicht

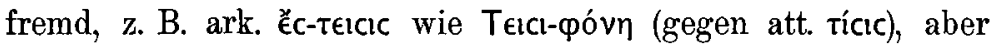
er findet sich hier weit seltener. Hier hat sich eben die nominale Natur dieser Substantiva in weit höherem Maße behauptet.

Was weiter die vidádvasu-Komposita betrifft, ein Typus, der nur im Arischen nachgewiesen ist, so denkt Jacobi S. 70 f. an zweierlei. Sie sollen entweder so entstanden sein, daß der Gebrauch von Formen wie *vida-vasu- das Sprachgefühl befremdet und man deshalb zur Verdeutlichung der Funktion des ersten Gliedes den schwachen Partizipialstamm eingeführt habe, oder vielleicht sei vidat in vidádvasu- ein Injunktiv, und das Kompositum habe ursprünglich 'er (oder. sie) möge Güter gewinnen" bedeutet. Gegen die letztere Auffassung läßt sich dasselbe einwenden, was Delbrück gegen die Erklärung von bháratu als bhárat $+u$ vorgebracht hat (s. oben S. 71). Sie ist aber auch schon darum wenig glaubhaft, weil auf Grund der 3. Person Sing. adhortativer Modi solche Komposita in den indogermanischen Sprachen sonst nicht erwachsen sind. Bei der ersteren Erklärung aber versteht man nicht, warum Formen wie *vida-vasu- das Sprachgefühl sollen befremdet haben.

Zunächst hat man wieder zuzusehen, ob nicht das erste Glied in den zahlreichen Formen mit -at- wie vidad-vasu- und

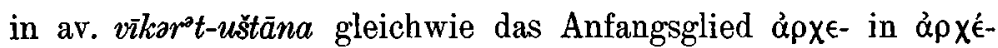
какос eine Formation war, die an und durch sich selbst eine imperativische Funktion hatte. Es liegt Anknüpfung an die In-

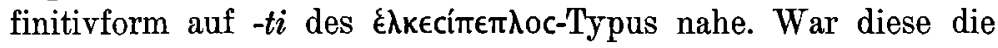
Stammform der ti-Abstrakta, so war das Vorderglied des vidádvasu-Typus möglicherweise die Stammform der $t$-Abstrakta, und war av. frā $a t i[-\bar{c} a]$ ' 'zu fördern', wie Bartholomae annimmt, ein

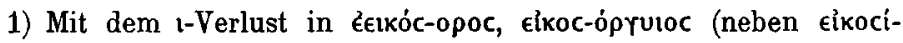
$\pi \eta \chi \cup c)$ hat es eine besondere Bewandtnis. Diese Formen stellten sich zu

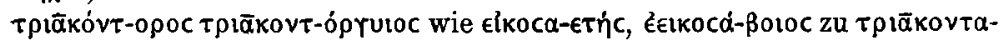
$\epsilon \tau \dot{n} c, \tau \rho t \bar{a} \kappa o v \tau d \dot{-z u Y o c}$. 
Lokativ auf $-i$, so könnten vidát- usw. flexionslose Lokative derselben Stammklasse gewesen sein.

Nach Ludwig Rigveda 6, 264 hätte das Vedische Infinitive auf -at gehabt, so daß man sich zunächst an sie halten könnte. Ludwig zählt als solche aus dem RV. auf: adat, išnat, gat, juşat, trpat, dršat, dravat, drahyat, dhršat, patayat, pibat, šravat, srjat (vgl. dazu seine Bemerkung zu RV. 10, 37, 11 in Bd. 4, 133). Leider sieht es aber mit der Gewähr der infinitivischen Funktion dieser Formen nicht zum besten aus. Von vornherein auszu-

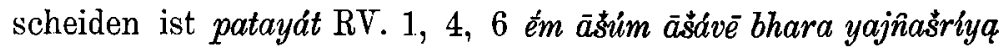
nґmádanam patayán mandayátsakham, was Ludwig übersetzt: 'bring (den Trank) heran, fliegen zu machen (daß er fliegen mache) den Freunderfreuer'. patayát ist vielmehr patayátsakham, d. h. man hat das letzte Glied des folgenden Kompositums zu ergänzen und demnach zu übersetzen 'den den Freund fliegen machenden (beflügelnden) und erfreuenden'. Ferner werden mit bestem Fug als adverbiale Akkusative des Part. Präs. von andern betrachtet dhrşăt $8,21,2$ 'kühnlich, herzhaft' (dazu der eben-

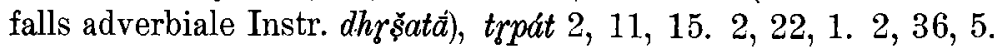
$3,32,2.7,56,10.10,116,1$ 'in genügender Weise, zur Genüge, bis zur Sättigung', dravát 8, 5, 7. Vāl. 1, 5 'eilend', drahyát 2, 11, 15 'tüchtig', dyugát 8, 86, 4 'zum Himmel gehend'. Schwierige Stellen, aber jedenfalls Stellen, denen keine Beweiskraft zugunsten von Ludwigs Ansicht inne wohnt, sind 1, 115, 5 (rúsat, s. Komment.), 4, 27, 3 (srját), 7, 32, 5 (šrávat), 8, 2, 23 (pibat) $10,61,5$ (iṣnát). Als besonders deutliche Infinitive bezeichnet Ludwig im Kommentar şját und yát 1, 174, 4, juş̌át 10, 20, 5, adát und pibat 10, 37, 11. Aber auch hier sind Bedenken gegen die Infinitivnatur nichts weniger als ausgeschlossen. Am ehesten scheint vielleicht jușát für Ludwig zu sprechen, der jușád dhavyáa mánuşasyōrdhvás tasthäv ł́bhvā yajné $e^{\prime}$ übersetzt ${ }^{\circ} z u$ genießen des Menschen Havya hat empor sich erhoben der Anstellige beim Opfer'. Wenn jedoch trpát teils Adverbium sein kann, teils sein muß, so wird auch juşát Adverbium sein, nur daß es einen Objektsakkusativ bei sich hat (vgl. Objektsakkusative beim Absolutivum auf -am, ferner má káména 'aus Liebe zu mir' u. dgl.). Wo das Partizipium auf -at nicht solches bezeichnet, was mit der Haupthandlung vor sich geht, sondern was beabsichtigt ist, was die Veranlassung zu der Haupthandlung ist, liegt derselbe Gebrauch vor, den 
auch das frei flektierte Part. Präs. im Vedischen zeigt, wie z. B.

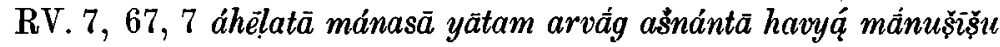
vikş̆u "kommt hierher mit gnädigem Sinne, um zu essen das Opfer in den menschlichen Wohnungen' (Delbrück Ai. Synt. 371, Vergl. Synt. 2, $478 \mathrm{f}$.).

Infinitive auf $-t$ sind somit für die Zeit des Sonderlebens der arischen Sprachen nicht verbürgt. Für die vorarische Zeit aber solche Infinitive vorauszusetzen, wäre bei dem Umstand, daß der vidádvasu-Typus nur im Arischen auftritt, äußerst kühn. So fragt es sich jetzt, ob dieser nicht erst durch eine urarische analogische Neuerung zustande gekommen ist.

Daß die Vorderglieder in den vinda- $x^{v} a r^{2} n a$-Formen den Ariern schon frühe als aktive Partizipia oder Nomina agentis erschienen, ist leicht begreiflich. Das Arische hatte Nominalstämme auf - $a$, die man ihrer Funktion nach als Präsenspartizipia bezeichnen darf, wie ai. vi-nayá- 'trennend', hinvá- 'anregend', pra-mrná- 'zerstörend', rērihá- 'leckend', av. par'sa- 'fragend', kar ${ }^{2} n t a-$ 'schneidend', srävaya- "hören lassend', da $\delta a$ - 'gebend" (Bartholomae KZ. 29, 557 ff.). Die Assoziation des Vorderglieds der vinda-xvarna-Komposita mit diesen war nahe gelegt, sobald uridg. $e$ und $o$ in $a$ zusammengefallen waren. Da nun neben jenen Partizipien auf $-a$ - (uridg. -o-) die auf $-n t$ - standen, z. B. pramrnánt- neben pramrná-, so konnten nun auch nt-Partizipia an die Stelle der alten Imperative in unsere Komposita eindringen. Ich stimme hiernach Jacobi (S. 71) darin bei, daß das Verschwinden der å $\rho \chi$ ékakoc-Formen im Indischen durch die Ausbreitung des vidádvasu-Typus hervorgerufen worden sei. Aber was war die Ursache dieser Ausbreitung auf Kosten des älteren Typus? Daß Formen wie *vidávasu- an sich anstößig gewesen sein sollten, ist schlechterdings nicht einzusehen. Irre ich nicht, so lag die Ursache in dem Vorhandensein von Bahuvrihi mit einem Partizipium als erstem Teil, wie ai. dravád-ašva-s 'mit eilenden Rossen' av. frao9at-aspa- 'mit schnaubenden Rossen' (daneben solche wie ai. šrút-karna- av. srut-gaoša- 'mit hörenden Ohren'). Es scheint diesen Komposita ähnlich gegangen zu sein, wie im Griechischen gewissen Bahuvrīhi mit adjektivischem Vorder-

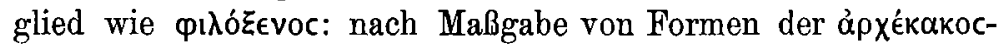
Klasse wurde $\varphi \imath \lambda \varepsilon_{\epsilon \in v o c}$ umgedeutet als 'den Gastfreund liebend' (was den Übergang von $\epsilon$ in $o$ in verbalen Komposita hervorrief

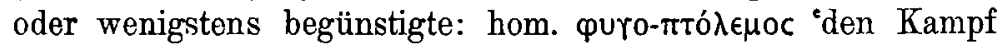


$76 \mathrm{~K}$. Brugmann, Zur Wortzusammensetzung in den idg. Sprachen.

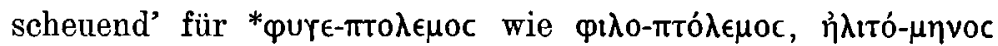

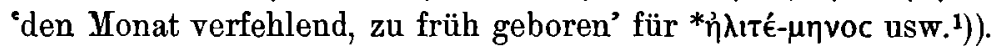
Eine solche Umwertung war im Arischen in solchen Fällen möglich, wo das Partizip ebenso gut als Attribut des hinteren Kompositionsglieds wie als Attribut des exozentrischen Subjekts aufgefaßt werden konnte. Etwa ein Kompositum mit der Bedeutung 'sich erkühnenden Sinn habend' (vgl. dhršan-manas-) konnte als 'sich erkühnend in seinem Sinne, mit seinem Sinne', eines mit der Bedeutung 'laufende, eilende Räder habend', vom Wagen gesagt (vgl. dravác-cakra-s), als 'eilend mit den Rädern' erscheinen u. dgl. m. Daß das Schlußglied im vidadvasu-Typus nicht immer akkusativisch abhängig war, zeigen sprhayád-varna-s 'nach Glanz strebend' mit dativischer, sâdád-yōni-ş 'im Schoße sitzend' mit lokativischer Kasusbeziehung. War so in einigen Fällen diese Umdeutung geschehen, so mochten leicht Bildungen wie vidád-vasu-š Fuß fassen und mochten durch sie dann im Indischen die älteren Formen wie *vidá-vasu-ş allmählich ganz verdrängt werden. Nur Formen ohne thematischen Vokal konnten von diesem Überwuchern des at-Typus nicht betroffen werden. Daher noch dídy-agni- und eventuell stháa-rašman- (S. 68 f.).

Av. vilkar ${ }^{\circ} t$-uštana- erklärt sich aus der nahen begrifflichen Verwandtschaft der mit - $t$ - gebildeten Adjektiva mit den $n t$ Partizipia.

Ist hiernach der vidádvasu-Typus von ganz anderer Entstehungsart als der dätivära-Typus, so fällt von jenem aus kein Licht auf die Frage, ob dāti- in dáti-vāra-der Lok. des Stammes dät- oder unflektierter Stamm auf $-i$ gewesen ist. Diese Frage muß ich offen lassen, sie ist indessen, wie schon oben bemerkt wurde, für uns nebensächlich.

Leipzig.

K. Brugmann.

1) Vgl. Jacobi S. 52 ff. - Außerdem vgl. die aisl. Komposita wie Hengiand-kiapta, Name eines Riesenweibs, "die den Unterkiefer hängen läßt', neben Hengi-kiapta 'Hängekiefer habend', Velland-katla, Name einer Quelle, 'Kesselkocher', die Falk PBrB. 14, 42 f. bespricht. Ob Falk die für die entwicklungsgeschichtliche Beurteilung dieser Partizipialkomposita in Betracht kommenden Momente alle angeführt hat, ist mir zweifelhaft. 